\title{
Volkov solution for two laser beams and ITER
}

\author{
Miroslav Pardy \\ Laboratory of the Plasma Physics \\ and \\ Department of Physical Electronics \\ Masaryk University \\ Kotlářská 2, 61137 Brno, Czech Republic \\ e-mail:pamir@physics.muni.cz
}

July 11, 2018

\begin{abstract}
We find the solutions of the Dirac equation for two plane waves (laser beams) and we determine the modified Compton formula for the scattering of two photons on an electron. The practical meaning of the two laser beams is, that two laser beams impinging on a target which is constituted from material in the form a foam, can replace 100-200 laser beams impinging on a normal target and it means that the nuclear fusion with two laser beams is realistic in combination with the thermonuclear reactor such as ITER.
\end{abstract}




\section{Introduction}

The application of a laser as a source of intense electromagnetic radiation enables to study the new class of physical processes which are running in the intense field of the electromagnetic wave. The probability of some processes are increased, or, decreased in comparison with the processes in vacuum. For instance the probability of the process $\pi \rightarrow \mu+\nu$ is increased and the probability of the process $\pi \rightarrow e+\nu$ is decreased. Some processes which are forbidden in vacuum, are allowed in the intense field of the electromagnetic wave $(\nu \rightarrow \pi+\mu$, or, $e \rightarrow \pi+\nu)$. The polarization of the electromagnetic wave plays important role.

The situation with the two electromagnetic wave is the next step and the future direction of the laser physics of elementary particles. The two laser beams can be used in the thermonuclear reactor instead of many laser beams. Two laser beams impinging on a target which is constituted from material in the form of foam, can replace 100-200 laser beams impinging on a normal target and it means that the nuclear fusion with two laser beams is realistic inside of the thermonuclear reactor such as ITER in Cadarache near Aix-en-Provence in France. ITER means "the way" in Latin and it will be prepared to produce $500 \mathrm{MW}$ of fusion power in 2016 .

The laser field, or, the two laser fields is also the detector of the new properties of elementary particles which cannot be revealed without laser. The situation with the two independent lasers can give evidently further information on the properties of elementary particles. At the same time the laser and the system of two lasers can be considered in chemistry as a specific catalyzer which was not known before the existence of the laser physics. So, laser methods in particle physics and chemistry can form in the near future the new scientific revolution, which is not described in the standard monographs on the scientific revolutions.

We consider here the electron described by the the Dirac equation for two different four-potentials of the plane electromagnetic waves. We derive the partial differential equation for the wave function, which is generalized form of the Volkov equation. We find the solutions of the Dirac equation for two orthogonal plane waves. We determine the modified Compton formula for the scattering of two photons on an electron.

The solution of the Dirac equation for the two waves was given by Sen Gupta (Sen Gupta, 1967) in the form of the Fourier series, however without immediate application. The solution of the Dirac equation for the two waves with the perpendicular polarization was given for instance by Lyulka $(1974,1975,1977,1985)$ who described the decay of particles in the two laser fields. The derivation of the two-wave solution is not explicitly involved in Lyulka articles. So,we investigate the situation and present our results.

To be pedagogically clear, we remind in the next section the derivation of the Volkov (1935) solution of the Dirac equation in vacuum.

\section{Volkov solution of the Dirac equation with massless photons}

We follow the method of derivation and metric convention of (Berestetzkii et al., 1989): 


$$
(\gamma(p-e A)-m) \psi=0
$$

where

$$
A^{\mu}=A^{\mu}(\varphi) ; \quad \varphi=k x .
$$

We suppose that the four-potential satisfies the Lorentz gauge condition

$$
\partial_{\mu} A^{\mu}=k_{\mu}\left(A^{\mu}\right)^{\prime}=\left(k_{\mu} A^{\mu}\right)^{\prime}=0,
$$

where the prime denotes derivative with regard to $\varphi$. From the last equation follows

$$
k A=\text { const }=0,
$$

because we can put the constant to zero. The tensor of electromagnetic field is

$$
F_{\mu \nu}=k_{\mu} A_{\nu}^{\prime}-k_{\nu} A_{\mu}^{\prime}
$$

Instead of the linear Dirac equation (1), we consider the quadratic equation, which we get by multiplication of the linear equation by operator $(\gamma(p-e A)+m)$ (Berestetzkii et al., 1989). We get:

$$
\left[(p-e A)^{2}-m^{2}-\frac{i}{2} e F_{\mu \nu} \sigma^{\mu \nu}\right] \psi=0 .
$$

Using $\partial_{\mu}\left(A^{\mu} \psi\right)=A^{\mu} \partial_{\mu} \psi$, which follows from eq. (3), and $\partial_{\mu} \partial^{\mu}=\partial^{2}=-p^{2}$, with $p_{\mu}=i\left(\partial / \partial x^{\mu}\right)=i \partial_{\mu}$, we get the quadratic Dirac equation for the four potential of the plane wave:

$$
\left[-\partial^{2}-2 i e(A \partial)+e^{2} A^{2}-m^{2}-i e(\gamma k)\left(\gamma A^{\prime}\right)\right] \psi=0 .
$$

We are looking for the solution of the last equation in the form:

$$
\psi=e^{-i p x} F(\varphi)
$$

After insertion of eq. (8) into eq. (7), we get with $\left(k^{2}=0\right)$

$$
\partial^{\mu} F=k^{\mu} F^{\prime}, \quad \partial_{\mu} \partial^{\mu} F=k^{2} F^{\prime \prime}=0,
$$

the following equation for $F(\varphi)$

$$
2 i(k p) F^{\prime}+\left[-2 e(p A)+e^{2} A^{2}-i e(\gamma k)\left(\gamma A^{\prime}\right)\right] F=0 .
$$

The integral of the last equation is of the form (Berestetzkii et al., 1989):

$$
F=\exp \left\{-i \int_{0}^{k x}\left[\frac{e(p A)}{(k p)}-\frac{e^{2}}{2(k p)} A^{2}\right] d \varphi+\frac{e(\gamma k)(\gamma A)}{2(k p)}\right\} \frac{u}{\sqrt{2 p_{0}}},
$$

where $u / \sqrt{2 p_{0}}$ is the arbitrary constant bispinor.

$\mathrm{Al}$ powers of $(\gamma k)(\gamma A)$ above the first are equal to zero, since 


$$
(\gamma k)(\gamma A)(\gamma k)(\gamma A)=-(\gamma k)(\gamma k)(\gamma A)(\gamma A)+2(k A)(\gamma k)(\gamma A)=-k^{2} A^{2}=0
$$

where we have used eq. (4) and relation $k^{2}=0$. Then we can write:

$$
\exp \left\{e \frac{(\gamma k)(\gamma A)}{2(k p)}\right\}=1+\frac{e(\gamma k)(\gamma A)}{2(k p)} .
$$

So, the solution is of the form:

$$
\psi_{p}=R \frac{u}{\sqrt{2 p_{0}}} e^{i S}=\left[1+\frac{e}{2 k p}(\gamma k)(\gamma A)\right] \frac{u}{\sqrt{2 p_{0}}} e^{i S},
$$

where $u$ is an electron bispinor of the corresponding Dirac equation

$$
(\gamma p-m) u=0
$$

and we shall take it to be normalized by condition $\bar{u} u=2 \mathrm{~m}$. The mathematical object $S$ is the classical Hamilton-Jacobi function, which was determined in the form:

$$
S=-p x-\int_{0}^{k x} \frac{e}{(k p)}\left[(p A)-\frac{e}{2} A^{2}\right] d \varphi .
$$

The current density is

$$
j^{\mu}=\bar{\psi}_{p} \gamma^{\mu} \psi_{p}
$$

where $\bar{\Psi}$ is defined as the transposition of (14), or,

$$
\bar{\psi}_{p}=\frac{\bar{u}}{\sqrt{2 p_{0}}}\left[1+\frac{e}{2 k p}(\gamma A)(\gamma k)\right] e^{-i S} .
$$

After insertion of $\Psi_{p}$ and $\bar{\Psi}_{p}$ into the current density, we have:

$$
j^{\mu}=\frac{1}{p_{0}}\left\{p^{\mu}-e A^{\mu}+k^{\mu}\left(\frac{e(p A)}{(k p)}-\frac{e^{2} A^{2}}{2(k p)}\right)\right\} .
$$

\section{The solution of the Dirac equation for two plane waves.}

We suppose that the total vector potential is given as a superposition of the potential $A$ and $B$ as follows:

$$
V_{\mu}=A_{\mu}(\varphi)+B_{\mu}(\chi)
$$

where $\varphi=k x$ and $\chi=\kappa x$ and $k \neq \kappa$.

We suppose that the Lorentz condition is valid. Or,

$$
\partial_{\mu} V^{\mu}=0=k_{\mu} \frac{\partial A^{\mu}}{\partial \varphi}+\kappa_{\mu} \frac{\partial B^{\mu}}{\partial \chi}=k_{\mu} A_{\varphi}^{\mu}+\kappa_{\mu} B_{\chi}^{\mu}
$$


where the subscripts $\varphi, \chi$ denote partial derivatives. The equation (21) can be written in the more simple form if we notice that partial differentiation with respect to $\varphi$ concerns only $A$ and partial differentiation with respect to $\chi$ concerns only $B$. So we write instead eq. (21).

$$
\partial_{\mu} V^{\mu}=0=k_{\mu}\left(A^{\mu}\right)^{\prime}+\kappa_{\mu}\left(B^{\mu}\right)^{\prime}=k A^{\prime}+\kappa B^{\prime} .
$$

Without loss of generality, we can write instead of equation (22) the following one

$$
k_{\mu}\left(A^{\mu}\right)^{\prime}=0 ; \quad \kappa_{\mu}\left(B^{\mu}\right)^{\prime}=0 ; \quad \text { or }, \quad k A=\text { const }=0 ; \quad \kappa B=\text { const }=0,
$$

putting integrating constant to zero.

The electromagnetic tensor $F_{\mu \nu}$ is expressed in the new variables as in (5)

$$
F_{\mu \nu}=k_{\mu} A_{\nu}^{\prime}-k_{\nu} A_{\mu}^{\prime}+\kappa_{\mu} B_{\nu}^{\prime}-\kappa_{\nu} B_{\mu}^{\prime} .
$$

Now, we can write Dirac equation for the two potentials the form

$$
\left[-\partial^{2}-2 i e(V \partial)+e^{2} V^{2}-m^{2}-\frac{i}{2} e F_{\mu \nu} \sigma^{\mu \nu}\right] \psi=0 .
$$

where $V=A+B, F_{\mu \nu}$ is given by eq. (24) and the combination of it with $\sigma$ is defined as follows:

$$
\frac{i}{2} e F_{\mu \nu} \sigma^{\mu \nu}=i e(\gamma k)\left(\gamma A^{\prime}\right)+i e(\gamma \kappa)\left(\gamma B^{\prime}\right)
$$

We will look for the solution in the standard Volkov form (8), or:

$$
\psi=e^{-i p x} F(\varphi, \chi) .
$$

After performing all operations prescribed in eq. (25), we get the following partial differential equation for the unknown function $F(\varphi, \chi)$ :

$$
\begin{gathered}
-2 k \kappa F_{\varphi \chi}+(2 i p k-2 i k B) F_{\varphi}+(2 i p \kappa-2 i e A \kappa) F_{\chi}+ \\
\left(e^{2}(A+B)^{2}-2 e(A+B) p-i e(\gamma k)\left(\gamma A_{\varphi}\right)-i e(\gamma \kappa)\left(\gamma B_{\chi}\right)\right) F=0 .
\end{gathered}
$$

\section{The solution of the Dirac equation for two orthog- onal waves}

The equation (28) was simplified by author (Pardy, 2004b) putting $k \kappa=0$. However, ignoring this simplification, we write eq. (28) in the following form:

$$
a F_{\varphi}+b F_{\chi}+c F=2 k \kappa F_{\varphi \chi}
$$

where

$$
a=2 i p k-2 i e k B ; \quad b=2 i p \kappa-2 i e \kappa A
$$


and

$$
c=e^{2}(A+B)^{2}-2 e(A+B) p-i e(\gamma k)\left(\gamma A^{\prime}\right)-i e(\gamma \kappa)\left(\gamma B^{\prime}\right) .
$$

and the term of with the two partial derivations is not present because of $k \kappa=0$.

For the field which we specify by the conditions

$$
k B=0 ; \quad \kappa A=0 ; \quad A B=0,
$$

we have:

$$
\begin{gathered}
2 i p k F_{\varphi}+2 i p \kappa \kappa F_{\chi}+e^{2} A^{2}+e^{2} B^{2}-2 e p A-2 e p B-i e(\gamma k)\left(\gamma A^{\prime}\right)- \\
\left.i e(\gamma \kappa)\left(\gamma B^{\prime}\right)\right) F=2 k \kappa F_{\varphi \chi} .
\end{gathered}
$$

Now, we are looking for the solution in the most simple form

$$
F(\varphi, \chi)=X(\varphi) Y(\chi)
$$

After insertion of (34) into (33) and division the new equation by $X Y$ we get the terms depending only on $\varphi$, and on $\chi$. Or, in other words we get:

$$
\begin{aligned}
& \left(2 i(p k+i k \kappa) \frac{X^{\prime}}{X}+e^{2} A^{2}-2 e p A-i e(\gamma k)\left(\gamma A^{\prime}\right)\right)+ \\
& \left(2 i(p \kappa+i k \kappa) \frac{Y^{\prime}}{Y}+e^{2} B^{2}-2 e p B-i e(\gamma \kappa)\left(\gamma B^{\prime}\right)\right)=0
\end{aligned}
$$

So, there are terms dependent on $\varphi$ and terms dependent on $\chi$ only in eq. (35). The only possibility is that they are equal to some constant $\lambda$ and $-\lambda$. Then,

$$
2 i(p k+i k \kappa) X^{\prime}+\left(e^{2} A^{2}-2 e p A-i e(\gamma k)\left(\gamma A^{\prime}\right)\right) X=\lambda X
$$

and

$$
2 i(p \kappa+i k \kappa) Y^{\prime}+\left(e^{2} B^{2}-2 e p B-i e(\gamma \kappa)\left(\gamma B^{\prime}\right)\right) Y=-\lambda Y
$$

We put $\lambda=0$ without lost of generality. Now, the solution of eq. (35) is reduced to the solution of two equations only. Because the form of the equations is similar to the form of eq. (14) we can write the solution of these equations as follows:

$$
X=\left[1+\frac{e}{2(k p+i k \kappa)}(\gamma k)(\gamma A)\right] \frac{u}{\sqrt{2 p_{0}}} e^{i S_{1}}
$$

with

$$
S_{1}=\int_{0}^{k x} \frac{e}{(k p+i k \kappa)}\left[(p A)-\frac{e}{2}(A)^{2}\right] d \varphi
$$

and 


$$
Y=\left[1+\frac{e}{2(\kappa p+i k \kappa)}(\gamma \kappa)(\gamma B)\right] \frac{u}{\sqrt{2 p_{0}}} e^{i S_{2}}
$$

with

$$
S_{2}=-\int_{0}^{\kappa x} \frac{e}{(\kappa p+i k \kappa)}\left[(p B)-\frac{e}{2}(B)^{2}\right] d \chi .
$$

The total solution is then of the form:

$$
\psi_{p}=\left[1+\frac{e}{2(k p+i k \kappa)}(\gamma k)(\gamma A)\right]\left[1+\frac{e}{2(\kappa p+i k \kappa)}(\gamma \kappa)(\gamma B)\right] \frac{u}{\sqrt{2 p_{0}}} e^{i\left(S_{1}(A)+S_{2}(B)\right)} .
$$

\section{The standard Compton process}

In order to find the wave function of the electron in the two laser beams, let us first remind the well known Compton problem following from the Volkov solution. The pioneering articles of this problem was written by Sen Gupta (1952) solving the Compton scattering in the strong magnetic field. The next application of the Volkov solution were done for the problem of pair production by collision between a strong and coherent electromagnetic field and a single energetic photon (Reiss, 1962). Later, Nikishov and Ritus (Nikishov

et al., 1964) and Goldman (1964a; 1964b) used the Reiss ideas in their articles. Plenty of problems concerning the application of the Volkov solution can be seen in the Ritus article (Ritus, 1979).

Let us consider electromagnetic monochromatic plane wave which is polarized in a circle. We write the four-potential in the form:

$$
A=a_{1} \cos \varphi+a_{2} \sin \varphi,
$$

where the amplitudes $a_{i}$ are equal in magnitude and orthogonal, or,

$$
a_{1}^{2}=a_{2}^{2}=a^{2}, \quad a_{1} a_{2}=0 .
$$

Then, it possible to show that the Volkov solution for this situation is of the form (Berestetzkii et al., 1989):

$$
\begin{gathered}
\psi_{p}=\left\{1+\left(\frac{e}{2(k p)}\right)\left[(\gamma k)\left(\gamma a_{1}\right) \cos \varphi+(\gamma k)\left(\gamma a_{2}\right) \sin \varphi\right]\right\} \frac{u(p)}{\sqrt{2 q_{0}}} \times \\
\exp \left\{-i e \frac{\left(a_{1} p\right)}{(k p)} \sin \varphi+i e \frac{\left(a_{2} p\right)}{(k p)} \cos \varphi-i q x\right\},
\end{gathered}
$$

where

$$
q^{\mu}=p^{\mu}-e^{2} \frac{a^{2}}{2(k p)} k^{\mu}
$$

follows from eq. (19) as a time-average value. In other words, $q^{\mu}$ is the mean value of quantity $p_{0} j^{\mu}$. 
We know that the matrix element $M$ corresponding to the emission of photon by an electron in the electromagnetic field is as follows (Berestetzkii et al., 1989):

$$
S_{f i}=-i e^{2} \int d^{4} x \bar{\psi}_{p^{\prime}}\left(\gamma e^{\prime *}\right) \psi_{p} \frac{e^{i k^{\prime} x}}{\sqrt{2 \omega^{\prime}}}
$$

where $\psi_{p}$ is the wave function of an electron before interaction with the laser photons and $\psi_{p^{\prime}}$ is the wave function of electron after emission of photon with components $k^{\prime \mu}=\left(\omega^{\prime}, \mathbf{k}^{\prime}\right)$. The quantity $e^{*}$ is the polarization four-vector of emitted photon.

The matrix element (47) involves the following linear combinations:

$$
\begin{gathered}
e^{-i \alpha_{1} \sin \varphi+i \alpha_{2} \cos \varphi} \\
e^{-i \alpha_{1} \sin \varphi+i \alpha_{2} \cos \varphi} \cos \varphi \\
e^{-i \alpha_{1} \sin \varphi+i \alpha_{2} \cos \varphi} \sin \varphi,
\end{gathered}
$$

where

$$
\alpha_{1}=e\left(\frac{a_{1} p}{k p}-\frac{a_{1} p^{\prime}}{k p^{\prime}}\right)
$$

and

$$
\alpha_{2}=e\left(\frac{a_{2} p}{k p}-\frac{a_{2} p^{\prime}}{k p^{\prime}}\right),
$$

Now, we can expand exponential function in the Fourier series, where the coefficients of the expansion will be $B_{s}, B_{1 s}, B_{2 s}$. So we write:

$$
\begin{gathered}
e^{-i \alpha_{1} \sin \varphi+i \alpha_{2} \cos \varphi}=e^{-i \sqrt{\alpha_{1}^{2}+\alpha_{2}^{2}} \sin \left(\varphi-\varphi_{0}\right)}=\sum_{s=-\infty}^{\infty} B_{s} e^{-i s \varphi} \\
e^{-i \alpha_{1} \sin \varphi+i \alpha_{2} \cos \varphi} \cos \varphi=e^{-i \sqrt{\alpha_{1}^{2}+\alpha_{2}^{2}} \sin \left(\varphi-\varphi_{0}\right)} \cos \varphi=\sum_{s=-\infty}^{\infty} B_{1 s} e^{-i s \varphi} \\
e^{-i \alpha_{1} \sin \varphi+i \alpha_{2} \cos \varphi} \sin \varphi=e^{-i \sqrt{\alpha_{1}^{2}+\alpha_{2}^{2}} \sin \left(\varphi-\varphi_{0}\right)} \sin \varphi=\sum_{s=-\infty}^{\infty} B_{2 s} e^{-i s \varphi}
\end{gathered}
$$

The Coefficients $B_{s}, B_{1 s}, B_{2 s}$ can be expressed by means of the Bessel function as follows (Berestetzkii et al., 1989):

$$
\begin{gathered}
B_{s}=J_{s}(z) e^{i s \varphi_{0}} \\
B_{1 s}=\frac{1}{2}\left[J_{s+1}(z) e^{i(s+1) \varphi_{0}}+J_{s-1}(z) e^{i(s-1) \varphi_{0}}\right] \\
B_{2 s}=\frac{1}{2 i}\left[J_{s+1}(z) e^{i(s+1) \varphi_{0}}-J_{s-1}(z) e^{i(s-1) \varphi_{0}}\right],
\end{gathered}
$$

where the quantity $z$ are now defined through the $\alpha$-components, or, 


$$
z=\sqrt{\alpha_{1}^{2}+\alpha_{2}^{2}}
$$

and

$$
\cos \varphi_{0}=\frac{\alpha_{1}}{z} ; \quad \sin \varphi_{0}=\frac{\alpha_{2}}{z} .
$$

Functions $B_{s}, B_{1 s}, B_{2 s}$ are related one to another as follows:

$$
\alpha_{1} B_{1 s}+\alpha_{2} B_{2 s}=s B_{s},
$$

which follows from the well known relation for Bessel functions:

$$
J_{s-1}(z)+J_{s+1}(z)=\frac{2 s}{z} J_{s}(z) .
$$

The matrix element (47) can be written in the form (Berestetzkii et al., 1989):

$$
S_{f i}=\frac{1}{\sqrt{2 \omega^{\prime} 2 q_{0} 2 q_{0}^{\prime}}} \sum_{s} M_{f i}^{(s)}(2 \pi)^{4} i \delta^{(4)}\left(s k+q-q^{\prime}-k^{\prime}\right),
$$

where the $\delta$-function involves the law of conservation:

$$
s k+q=q^{\prime}+k^{\prime} ; \quad s=1,2,3, \ldots
$$

with the relation

$$
q^{2}=q^{2}=m^{2}\left(1+\xi^{2}\right) \equiv m_{*}^{2}=m^{2}\left(1-\frac{e^{2} a^{2}}{m^{2}}\right),
$$

as it follows from eq. (46).

For $s=1$, eq. (64) has the physical meaning of the conservation of energy- momentum of the one-photon Compton process, $s=2$ has meaning of the two-photon Compton process and $s=n$ has meaning of the multiphoton interaction with $n$ photons.

It is possible to show, that the differential probability per unit volume and unit time of the emission of the $s$ harmonics is of the following form (Berestetzkii et al., 1989):

$$
d W_{s}=\left|M_{f i}^{(s)}\right|^{2} \frac{d^{3} k^{\prime} d^{3} q^{\prime}}{(2 \pi)^{6} 2 \omega^{\prime} 2 q_{0} 2 q_{0}^{\prime}}(2 \pi)^{4} \delta^{(4)}\left(s k+q-q^{\prime}-k^{\prime}\right) .
$$

In order to obtain the probability of emission of photon, we must make some operation with the matrix element $M$. We will here not perform these operations. We concentrate our attention on the conservation law in the formula (66). It can be expressed by words as follows. The multiphoton object with the momentum sk interacts with the electron of the momentum $q$, and the result is the electron with the momentum $q^{\prime}$ and one photon with the momentum $k^{\prime}$.

Now, let us consider the equation (64) in the form

$$
s k+q-k^{\prime}=q^{\prime} .
$$

If we introduce the angle $\Theta$ between $\mathbf{k}$ and $\mathbf{k}^{\prime}$, then, with $|\mathbf{k}|=\omega$ and $\left|\mathbf{k}^{\prime}\right|=\omega^{\prime}$, we get from the squared equation (67) in the rest system of electron, where $q=\left(m_{*}, 0\right)$, the following equation: 


$$
s \frac{1}{\omega^{\prime}}-\frac{1}{\omega}=\frac{s}{m_{*}}(1-\cos \Theta),
$$

which is modification of the original equation for the Compton process

$$
\frac{1}{\omega^{\prime}}-\frac{1}{\omega}=\frac{1}{m}(1-\cos \Theta) \text {. }
$$

So, we see that Compton effect described by the Volkov solution of the Dirac equation differs from the original Compton formula only by the existence of the renormalized mass and parameter $s$ of the multiphoton interaction.

We know that the last formula of the original Compton effect can be written in the form suitable for the experimental verification, namely:

$$
\lambda^{\prime}-\lambda=\Delta \lambda=4 \pi \frac{\hbar}{m c} \sin ^{2} \frac{\Theta}{2},
$$

which was used by Compton for the verification of the quantum nature of light.

\section{The two-photon Compton process}

In case of the two laser beams which are not collinear the experimental situation involves possibility that the two different photons can interact with one electron. The theory does not follow from the standard one-photon Volkov solution because in the standard approach the multiphoton interaction involve the collinear photon s and not photons from the two different lasers. The problem was solved by Lyulka in 1974 for the case of the two linearly polarized waves (Lyulka, 1974)

$$
A=a_{1} \cos \varphi ; \quad B=a_{2} \cos (\chi+\delta)
$$

with the standard conditions for $\varphi, \chi, k, \kappa$. The quantity $\delta$ is the phase shift.

The two-wave Volkov solution is given by eq. (42) and the matrix elements and appropriate ingredients of calculations are given by the standard approach as it was shown by Lyulka (Lyulka, 1974).

It was shown (Lyulka, 1974), that

$$
q^{\mu}=p^{\mu}-e^{2} \frac{a_{1}^{2}}{2(k p)} k^{\mu}-e^{2} \frac{a_{2}^{2}}{2(\kappa p)} \kappa^{\mu}
$$

and

$$
m_{*}^{2}=m^{2}\left(1-\frac{e^{2} a_{1}^{2}}{m^{2}}-\frac{e^{2} a_{2}^{2}}{m^{2}}\right) .
$$

The matrix element involves the extended law of conservation. Namely:

$$
s k+t \kappa+q=q^{\prime}+k^{\prime}+\kappa^{\prime},
$$

where $s$ and $t$ are natural numbers and the interpretation of the last equation is evident. The multiphoton objects with momenta $s k$ and $t \kappa$ interact with electron with momentum 
q. After interaction the electron has a momentum $q^{\prime}$ and two photons are emitted with momenta $k^{\prime}$ and $\kappa^{\prime}$.

Instead of equation (74), we can write

$$
s k+q-k^{\prime}=q^{\prime}+\kappa^{\prime}-t \kappa .
$$

From the squared form of the last equation and after some modification, we get the following generalized equation of the double Compton process for $s=t=1$ :

$$
\frac{1}{\omega^{\prime}}-\frac{1}{\omega}=\frac{1}{m_{*}}(1-\cos \Theta)+\frac{\Omega^{\prime}-\Omega}{\omega \omega^{\prime}}-\frac{\Omega \Omega^{\prime}}{\omega \omega^{\prime} m_{*}}(1-\cos \Xi),
$$

where the angle $\Xi$ is the angle between the 3 -momentum of the $\kappa$-photon and the 3 momentum of the $\kappa^{\prime}$-photon with frequency $\Omega$ and $\Omega^{\prime}$ respectively.

Let us remark that if the frequencies of the photons of the first wave substantially differs from the frequency of photons of the second electromagnetic wave, then, the derived formula (76) can be experimentally verified by the same way as the original Compton formula. To our knowledge, formula (76) is not involved in the standard textbooks on quantum electrodynamics because the two laser physics is at present time not sufficiently developed.

\section{Discussion}

We have discussed the problem of the Dirac equation with the two-wave potentials of the electromagnetic fields. While the Volkov solution for one potential is well known for long time, the case with the two waves represents the new problem. To our knowledge, the Compton process with two beams was not investigated experimentally by any laboratory.

This article is in a some sense author's continuation of the problems where the Compton, or Volkov solution plays substantial role (Pardy, 1998; 2001; 2003; 2004a; 2004b).

It is possible to consider the situation with sum of $\mathrm{N}$ waves, or,

$$
V=\sum_{i=1}^{N} A_{i}\left(\varphi_{i}\right) \quad \varphi_{i}=k_{i} x .
$$

The problem has obviously physical meaning because the problem of the laser compression of target by many beams is one of the prestigious problems of the today laser physics. The goal of the experiments is to generate the physical process of implosion. When an intense petawat laser light is uniformly impinged on a spherical fuel pellet,the laser energy is absorbed on the surface to generate a high-temperature plasma of 2-3 keV and an extremely high pressure of a few hundred megabars is generated. The pressure accelerates the outer shall of the target towards the target centre. If the dynamics is sufficiently spherically symmetric, the central area is heated up to 5-10 keV and fusion reaction starts (Nakai and Mima, 2004). The solution of that problem in the general form is not elementary and can be solved only by some laser institution such as the Lebedev institution of physics, the Livermore laser national laboratory and so on.

On the other hand, if the target is constituted from material in the form a foam, then instead of using 100-200 laser beams it is possible to use only two laser beams, as it is 
supposed in our article. Then the nuclear fusion is more realistic in the situation of the thermonuclear reactor (Rozanov, 2004).

Nuclear fusion involves the bringing together of atomic nuclei. The sum of the individual masses of the nucleons is greater than the mass of the whole nucleus. This is because the strong nuclear force holds the nucleons together. Then, the combined nucleus is a lower energy state than the nucleons separately. The energy difference is released in the fusion process.

There are two major fusion processes. The magnetic confinement and inertial confinement. The inertial fusion occurs inside targed fuel pellets by imploding them with laser or particle beam irradiation in brief pulses. It produces extremely high densities in the targed where the laser pulse creates a schock wave in the pellet that it intensified by its internal geometry. On the other hand, magnetic fusion devices, like the tokamak, operate at lower densities, but use magnetic fields to confine the plasma for longer time.

To achieve a burning plasma, a sufficiently high density of fuel must be heated to temperatures about 100 million degrees of Celsius that the nuclei collide often enough despite their natural repulsive forces and energy losses.

The fuels to be used are two isotopes of hydrogen. Namely, deuterium and tritium. While deuterium occurs naturally in sea water and it means it is inexhaustible, tritium can be bred in a fusion system when the light element, lithium, absorbs neutrons produced in the fusion reaction. World resources of lithium are inexhaustible and it means that also the energy obtained by fusion process is practically infinite.

\section{References}

Berestetzkii, V. B., Lifshitz, E. M. and Pitaevskii, L. P. (1989). Quantum Electrodynamics, Moscow, Nauka, (in Russian).

Goldman, I. I. (1964a). Intensity effects in Compton scattering, Zh. Exp. Teor. Fiz. 46, 1412; ibid. (1964b). Intensity effects in Compton scattering, Phys. Lett. 8(2), 103.

Lyulka, V. A. (1974). Quantum effects in intense electromagnetic fields, Sov. Phys. JETP 40, 815 .

Lyulka, V. A. (1975). Decays of elementary particles in the field of of the intense electromagnetic wave, Zh. Exp. Teor. Fiz. 69, Vol. 3(9), 800.

Lyulka, V. A. (1977). Quantum effects in the field of the nonchromatical electromagnetic wave, Zh. Exp. Teor. Fiz. 72, Vol. 3, 865.

Lyulka, V. A. (1985). Weak processes in the intense Electron in the ultrashort laser pulse electromagnetic fields, Journal of Nuclear Physics Vol. 5(11), 1211.

Nakai, S. and Mima, K. (2004). Laser driven inertial fusion energy: present and perspective, Rep. Prog. Phys. 67, 321.

Nikishov, A. I. and Ritus, V. I. (1964). Quantum processes in the field of electromagnetic 
wave in the constant field I., Zh. Eksp. Teor. Fiz. 46, 776.

Pardy, M. (1998). The quantum field theory of laser acceleration, Physics Letters A 243, 223.

Pardy, M. (2001). The quantum electrodynamics of laser acceleration, Radiation Physics and Chemistry 61, 321.

Pardy, M. (2003). Electron in the ultrashort laser pulse, International Journal of Theoretical Physics 42(1), 99.

Pardy, M. (2004a). Massive photons and the Volkov solution, International Journal of Theoretical Physics 43(1), 127.

Pardy, M. (2004b). Volkov solution for an electron in two wave fields, e-print hepph/0408288.

Reiss, H. R. (1962). Absorption of light by light, J. Math. Phys. 3, 59.

Rozanov, V. B. (2004). On the possible realization of spherical compression for fusion targets irradiated by two laser beams, Uspekhi Fiz. Nauk, 174(4), 371. (in Russian).

Ritus, V. I. (1979). The quantum effects of the interaction of elementary particles with the intense electromagnetic field, Trudy FIAN 111, 5.

Sen Gupta, N. D. (1952). Bull. Calcutta Math. Soc., 39, 147; ibid. 44, 175.

Sen Gupta, N. D. (1967). On the solution of the Dirac equation in the field of two beams of electromagnetic radiation, Zeitschrift für Physik 200, 13.

Volkov, D. M. (1935). Über eine Klasse von Lösungen der Diracschen Gleichung, Zeitschrift für Physik 94, 250. 